\title{
A NOTE ON DISCRETE MAXIMAL REGULARITY FOR FUNCTIONAL DIFFERENCE EQUATIONS WITH INFINITE DELAY
}

\author{
CLAUDIO CUEVAS AND CLAUDIO VIDAL
}

Received 4 October 2005; Accepted 1 November 2005

Dedicated to Juan Cuevas Gonzalez

Using exponential dichotomies, we get maximal regularity for retarded functional difference equations. Applications on Volterra difference equations with infinite delay are shown.

Copyright (c) 2006 C. Cuevas and C. Vidal. This is an open access article distributed under the Creative Commons Attribution License, which permits unrestricted use, distribution, and reproduction in any medium, provided the original work is properly cited.

\section{Introduction}

The maximal regularity problem for the discrete time evolution equations has been recently considered by Blunck $[4,5]$. Discrete maximal regularity properties appears not to be considered in the literature before the paper [5]. The continuous maximal regularity problem for time evolution equations is well-know (see $[1,4,5,19,20]$ and the reference contained therein).

In the present paper we are concerned in the study of maximal regularity for the following homogeneous retarded linear functional equation:

$$
x(n+1)=L\left(n, x_{n}\right), \quad n \geq n_{0} \geq 0,
$$

where $L: \mathbb{N}\left(n_{0}\right) \times \mathscr{B} \rightarrow \mathbb{C}^{r}$ is a bounded linear map with respect to the second variable, $\mathscr{B}$ denotes an abstract phase space that we will explain briefly later (for the basic theory of phase spaces, the reader is referred to the book by Hino et al. [14]); $x$. denotes the $\mathscr{B}$-valued function defined by $n \rightarrow x_{n}$, and $\mathbb{N}\left(n_{0}\right)$ denotes the set $\left\{n \in \mathbb{N} / n \geq n_{0}\right\}$.

The abstract phase spaces was introduced by Hale and Kato [13] for studying qualitative theory of functional differential equations with unbounded delay. The idea of considering phase spaces for studying qualitative properties of functional difference equations was used first by Murakami [18] for study some spectral properties of the solution operator for linear Volterra difference systems and then by Elaydi et al. [12] for study asymptotic equivalence of bounded solutions of a homogeneous Volterra difference system and its perturbation. 
Besides its theoretical interest, the study of abstract retarded functional difference equations in phase space has great importance in applications. For these reasons from then the theory of functional difference equations with infinite delay has drawn the attention of several authors (see $[6-12,16-18]$ ). The fact that the state space for functional difference equations is infinite dimensional require the development of methods and techniques from functional analysis. Questions concerning boundedness, convergence and asymptotic behavior of perturbations (1.1) has been studied by Cuevas [6], Cuevas and Pinto [8-10], Cuevas and Vidal [11], Cuevas and Del Campo [7]. Recently, a very interesting article has been published by Matsunaga and Murakami [16] concerning to the existence of the local stable manifolds, together with the local unstable manifolds and the local center-unstable manifolds for nonlinear autonomous functional difference equations in phase spaces. The results in [16] are based on a representation formula for solutions of nonhomogeneous linear functional difference equations. As application of the general results given in [16] have been obtained some results on stabilities and instabilities for the zero solution of equation $x(n+1)=L\left(x_{n}\right)+f\left(x_{n}\right)$, where $L: \mathscr{B} \rightarrow \mathbb{C}^{r}$ is a bounded linear operator and $f \in C^{1}\left(\mathscr{B}, \mathbb{C}^{r}\right)$ with $f(0)=f^{\prime}(0)=0$.

This paper deals with maximal regularity for functional difference equation with infinite delay under the hypothesis that the solution operator (1.1) has an exponential dichotomy. The problem of deciding when a functional difference equation has a such dichotomy is a priori much more complicated than for ordinary difference systems, because it is necessary to construct suitable projections, a wrong choice of projections would clearly cause very serious problems. Until now there is not a method to construct projections. In this work we show how one can generating projections (see Remark 4.5).

This paper is organized as follows. The second section provides the definitions and notations to be used in the results stated and proved in this work. In the third section, we study the maximal regularity problem for (1.1); while in the fourth section, we present applications to discrete Volterra difference equations with infinite delay. During the last few years discrete Volterra equations have emerged vigorously in several applied fields and there is much interest in developing the qualitative theory for such equations (see [15] for discussion and references).

\section{Preliminaries and notations}

Here we explain some notations and the phase space notion. As usual, we denote $\mathbb{Z}, \mathbb{Z}^{+}$ and $\mathbb{Z}^{-}$the set of all integers, the set of all nonnegative integers and the set of all nonpositive integers, respectively. Let $\mathbb{C}^{r}$ be the $r$-dimensional complex Euclidean space with norm $|\cdot|$. For any function $x: \mathbb{Z} \rightarrow \mathbb{C}^{r}$ and $n \in \mathbb{Z}$, we define the functions $x_{n}: \mathbb{Z}^{-} \rightarrow \mathbb{C}^{r}$ by $x_{n}(s)=x(n+s)$ for $s \in \mathbb{Z}^{-}$. We follow the terminology used in Murakami [17] thus the phase space $\mathscr{B}=\mathscr{H}\left(\mathbb{Z}^{-}, \mathbb{C}^{r}\right)$ is a Banach space (with norm denoted by $\|\cdot\|_{\mathscr{B}}$ ) which is a subfamily of functions from $\mathbb{Z}^{-}$into $\mathbb{C}^{r}$ and it is assumed to satisfy the following axioms.

(A) There are a positive constant $J>0$ and nonnegative functions $N(\cdot)$ and $M(\cdot)$ on $\mathbb{Z}^{+}$with the property that if $x: \mathbb{Z} \rightarrow \mathbb{C}^{r}$ is a function such that $x_{0} \in \mathscr{B}$, then for all $n \in \mathbb{Z}^{+}$, the following conditions are held.

(i) $x_{n} \in \mathscr{B}$.

(ii) $J|x(n)| \leq\left\|x_{n}\right\|_{\Re} \leq N(n) \operatorname{Sup}_{0 \leq s \leq n}|x(s)|+M(n)|| x_{0} \|_{\Re}$. 
(B) The inclusion map $i:\left(B\left(\mathbb{Z}^{-}, \mathbb{C}^{r}\right),\|\cdot\|_{\infty}\right) \rightarrow\left(\mathscr{B},\|\cdot\|_{\mathscr{B}}\right)$ is continuous, that is, there is a constant $K \geq 0$ such that $\|\varphi\|_{\mathscr{B}} \leq K\|\varphi\|_{\infty}$, for all $\varphi \in B\left(\mathbb{Z}^{-}, \mathbb{C}^{r}\right)\left(\right.$ where $B\left(\mathbb{Z}^{-}, \mathbb{C}^{r}\right)$ represents the bounded functions on $\mathbb{Z}^{-}$in $\mathbb{C}^{r}$ ).

From now on $\mathscr{S}$ will denote a phase space satisfying the axioms (A) and (B). For any $n \geq \tau$, we define the operator $T(n, \tau): \mathscr{B} \rightarrow \mathscr{B}$ by $T(n, \tau) \varphi=x_{n}(\tau, \varphi, 0)$ for $\varphi \in \mathscr{B}$, where $x(\cdot, \tau, \varphi, 0)$ denotes the solution of the homogeneous linear system (1.1) passing through $(\tau, \varphi)$. It is clear that the operator $T(n, \tau)$ is linear and by virtue of axiom (A) it is bounded on $\mathscr{B}$ and satisfies the following properties:

$$
T(n, s) T(s, \tau)=T(n, \tau) \quad \text { for } n \geq s \geq \tau, \quad T(n, n)=I \quad \text { for } n \geq n_{0} .
$$

The operator $T(n, \tau)$ is called the solution operator of the homogeneous linear system (1.1) (see [17] for details).

Definition 2.1. We say that system (1.1) has an exponential dichotomy on $\mathbb{N}\left(n_{0}\right)$ with data $(\alpha, \widetilde{K}, P(n))$ if $\alpha, \widetilde{K}$ are positive numbers and $P(n)$ are projectors in $\mathscr{B}$, such that if $Q(n)=I-P(n)$, then the following holds.

(i) $T(n, \tau) P(\tau)=P(n) T(n, \tau), n \geq \tau$.

(ii) The restriction $T(n, \tau) \mid \operatorname{Range}(Q(\tau)), n \geq \tau$, is an isomorphism of $\operatorname{Range}(Q(\tau))$ onto Range $(Q(n))$ and we define $T(\tau, n)$ as the inverse mapping.

(iii) $|T(n, \tau) P(\tau)| \leq \widetilde{K} e^{-\alpha(n-\tau)}, n \geq \tau$.

(iv) $|T(n, \tau) Q(\tau)| \leq \widetilde{K} e^{\alpha(n-\tau)}, \tau>n$.

The number $-\alpha$ limits the exponential growth of solutions in forward direction when started in Range $(P(\tau))$ correspondingly, $\alpha$ limits the exponential growth in backward direction when started in Range $(Q(\tau))$. Note that in the case $\alpha=0$ we have an ordinary dichotomy (see [11, Remark 2.1] for more details).

In what follows, we consider the $r \times r$ matrix function, $E^{0}(t), t \in \mathbb{Z}^{-}$, defined by

$$
E^{0}(t)= \begin{cases}I(r \times r \text { unit matrix }) & \text { if } t=0, \\ 0(r \times r \text { zero matrix }) & \text { if } t<0 .\end{cases}
$$

On the other hand, $\Gamma(n, s)$ denotes the Green function associated with (1.1), that is,

$$
\Gamma(n, s)= \begin{cases}T(n, s+1) P(s+1) & \text { if } n-1 \geq s, \\ -T(n, s+1) Q(s+1) & \text { if } s>n-1 .\end{cases}
$$

Definition 2.2. We say that system (1.1) has a discrete maximal regularity if for each $h \in$ $\ell^{p}\left(\mathbb{N}\left(n_{0}\right), \mathbb{C}^{r}\right)$ (with $\left.1 \leq p \leq+\infty\right)$ and each $\varphi \in P\left(n_{0}\right) \mathscr{B}$ the solution $z$ of the boundary value problem,

$$
\begin{gathered}
z(n+1)=L\left(n, z_{n}\right)+h(n), \quad n \geq n_{0}, \\
P\left(n_{0}\right) z_{n_{0}}=\varphi
\end{gathered}
$$

satisfies $\Delta z . \in \ell^{p}\left(\mathbb{N}\left(n_{0}\right), \mathscr{B}\right)$ (i.e, $\left.z . \in \mathcal{W}^{1, p}\right)$, where $\Delta$ is the difference operator of the first order. 
4 Discrete maximal regularity

\section{Maximal regularity for retarded functional difference equations}

We get the following result about maximal regularity of (1.1).

Theorem 3.1. Assume that system (1.1) has an exponential dichotomy on $\mathbb{N}\left(n_{0}\right)$ with data $(\alpha, \tilde{K}, P(n))$. Then, for any $h \in \ell^{P}\left(\mathbb{N}\left(n_{0}\right)\right)$ (with $\left.1 \leq p \leq+\infty\right)$ and any $\varphi \in \operatorname{Range}\left(P\left(n_{0}\right)\right)$, the boundary value problem (2.4) has a unique solution $z \in W^{1, p}\left(\mathbb{N}\left(n_{0}\right)\right)$, namely $z=z^{s p}+$ $z^{\text {hom }}$, where

$$
z_{n}^{s p}=\sum_{s=n_{0}}^{\infty} \Gamma(n, s) E^{0}(h(s)), \quad z_{n}^{\text {hom }}=T\left(n, n_{0}\right) P\left(n_{0}\right) \varphi .
$$

This solution $z$ satisfies $z \in \ell^{p^{\prime}}\left(\mathbb{N}\left(n_{0}\right)\right)$ for all $1 \leq p \leq p^{\prime} \leq+\infty$, and the following estimates hold:

$$
\begin{gathered}
\left(1-e^{-\alpha}\right)^{1-1 / p+1 / p^{\prime}}\left\|z^{s p}\right\|_{p^{\prime}}+\left(1-e^{-\alpha}\right)^{1-1 / p}\left\|z_{n_{0}}^{s p}\right\|_{\mathscr{B}} \leq 4 K \tilde{K}\|h\|_{p}, \\
\left(1-e^{-\alpha}\right)^{1 / p^{\prime}}\left\|z^{\text {hom }}\right\|_{p^{\prime}}+\left\|z_{n_{0}}^{\text {hom }}\right\|_{\mathscr{B}} \leq(\tilde{K}+1)\|\varphi\|_{\mathscr{B}} .
\end{gathered}
$$

In particular, if $p=+\infty$, we get

$$
\begin{gathered}
\left(1-e^{-\alpha}\right)\left[\left\|z^{s p}\right\|_{\infty}+\left\|z_{n_{0}}^{s p}\right\|_{\mathscr{B}}\right] \leq 4 K \tilde{K}\|h\|_{\infty}, \\
\left\|z^{\text {hom }}\right\|_{\infty}+\left\|z_{n_{0}}^{\text {hom }}\right\|_{\mathscr{B}} \leq(\tilde{K}+1)\|\varphi\|_{\Re} .
\end{gathered}
$$

Proof. The proof based on the Beyn and Lorenz's ideas contained on the argument of proof of [3, Theorem A.2]. Initially we will treat the existence problem. We observe that

$$
\begin{aligned}
T\left(n, n_{0}\right) & z_{n_{0}}+\sum_{s=n_{0}}^{n-1} T(n, s+1) E^{0}(h(s)) \\
= & T\left(n, n_{0}\right) P\left(n_{0}\right) \varphi-\sum_{s=n_{0}}^{\infty} T(n, s+1) Q(s+1) E^{0}(h(s)) \\
& +\sum_{s=n_{0}}^{n-1} T(n, s+1) E^{0}(h(s))=T\left(n, n_{0}\right) P\left(n_{0}\right) \varphi-\sum_{s=n_{0}}^{n-1} T(n, s+1) E^{0}(h(s)) \\
& +\sum_{s=n_{0}}^{n-1} \Gamma(n, s) E^{0}(h(s))+\sum_{s=n}^{\infty} \Gamma(n, s) E^{0}(h(s)) \\
& +\sum_{s=n_{0}}^{n-1} T(n, s+1) E^{0}(h(s))=z_{n} .
\end{aligned}
$$

Hence, from [11, Lemma 2.8], we get that $z=z^{s p}+z^{\text {hom }}$ solves the boundary value problem (2.4). Moreover, we can infer that $z$ is bounded. In fact, clearly $z^{\text {hom }}$ is bounded on $\mathbb{N}\left(n_{0}\right)$. On the other hand, we can get that

$$
\left\|z^{s p}\right\|_{\infty} \leq 2 K \tilde{K}\|h\|_{p}\left(1-e^{-\alpha}\right)^{1 / p-1} .
$$


To prove the uniqueness we use the crucial Murakami's representation formula (see, [17, Theorem 2.1, page 1155]) and the Beyn and Lorenz's uniqueness argument in a similar manner like in [2, Theorem A.1]. Indeed, let $y^{1}$ and $y^{2}$. be two bounded solutions of the boundary value problem (2.4). Put $z_{n}=y_{n}^{1}-y_{n}^{2}$, so $z(n)$ is solution of

$$
\begin{gathered}
x(n+1)=L\left(n, x_{n}\right), \quad n \geq n_{0}, \\
P\left(n_{0}\right) z_{n_{0}}=0 .
\end{gathered}
$$

Using the Murakami's representation formula we get that

$$
z_{n}=T\left(n, n_{0}\right) z_{n_{0}}, \quad n \geq n_{0}
$$

Now, by the property (ii) of Definition 2.1, we get

$$
z_{n_{0}}=T\left(n_{0}, n\right) Q(n) z_{n}, \quad n \geq n_{0}
$$

Then,

$$
\left\|z_{n_{0}}\right\|_{\mathscr{B}} \leq \tilde{K} e^{\alpha\left(n_{0}-n\right)}\|z \cdot\|_{\infty}, \quad n \geq n_{0}
$$

We conclude $z_{n_{0}}=0$, and hence $z_{n}=0$. Concluding the uniqueness.

We can verify that $z . \in \ell^{P}\left(\mathbb{N}\left(n_{0}\right)\right)$. It follows from the following estimates:

$$
\begin{gathered}
\left\|z^{s p}\right\|_{p} \leq 2 K \tilde{K}\|h\|_{p} /\left(1-e^{-\alpha}\right), \\
\left\|z^{\text {hom }}\right\|_{p} \leq \tilde{K}\left(1-e^{-\alpha}\right)^{-1 / p}\|\varphi\|_{\mathscr{B}} .
\end{gathered}
$$

Next, we will prove that the estimates (3.2) and (3.3). Let $p$ and $q$ be conjugated exponents. We have the following estimates:

$$
\begin{aligned}
\left\|z_{n}^{s p}\right\|_{\Re}^{p^{\prime}} & \leq(K \tilde{K})^{p^{\prime}}\left(\frac{2}{1-e^{-\alpha}}\right)^{p^{\prime} / q}\left(\sum_{s=n_{0}}^{\infty} e^{-\alpha|n-(s+1)|}|h(s)|^{p}\right)^{p^{\prime} / p} \\
& \leq(K \tilde{K})^{p^{\prime}}\left(\frac{2}{1-e^{-\alpha}}\right)^{p^{\prime} / q}\|h\|_{p}^{p^{\prime}-p} \sum_{s=n_{0}}^{\infty} e^{-\alpha|n-(s+1)|}|h(s)|^{p} .
\end{aligned}
$$

Then,

$$
\begin{aligned}
\left\|z^{s p}\right\|_{p^{\prime}}^{p^{\prime}} & \leq(K \tilde{K})^{p^{\prime}}\left(\frac{2}{1-e^{-\alpha}}\right)^{p^{\prime} / q}\|h\|_{p}^{p^{\prime}-p} \sum_{s=n_{0}}^{\infty}\left(\frac{2}{1-e^{-\alpha}}\right)|h(s)|^{p} \\
& \leq\left(2 K \tilde{K}\left(1-e^{-\alpha}\right)^{-1 / p^{\prime}-1 / q}\|h\|_{p}\right)^{p^{\prime}} .
\end{aligned}
$$

For the second term on the left-hand side of (3.2) we obtain

$$
\left\|z_{n_{0}}^{s p}\right\|_{\mathscr{B}}^{p^{\prime}} \leq(K \tilde{K})^{p^{\prime}}\left(\sum_{s=n_{0}}^{\infty} e^{-\alpha\left|n_{0}-(s+1)\right|}|h(s)|\right)^{p^{\prime}} \leq\left(2 K \tilde{K}\left(1-e^{-\alpha}\right)^{-1 / q}\|h\|_{p}\right)^{p^{\prime}} .
$$


Finally, we sum

$$
\left\|z_{n}^{\text {hom }}\right\|_{\mathscr{乃}}^{p^{\prime}} \leq \widetilde{K}^{p^{\prime}} e^{-\alpha p^{\prime}\left(n-n_{0}\right)}\|\varphi\|_{\mathscr{乃}}^{p^{\prime}}
$$

with respect to $n$ and find

$$
\left\|z^{\text {hom }}\right\|_{p^{\prime}} \leq \tilde{K}\left(1-e^{-\alpha p^{\prime}}\right)^{-1 / p^{\prime}}\|\varphi\|_{\Re} \leq \tilde{K}\left(1-e^{-\alpha}\right)^{-1 / p^{\prime}}\|\varphi\|_{\Re} .
$$

This leads to the desired estimate (3.3). This complete the proof of Theorem 3.1.

Remark 3.2. In [3], Beyn and Lorenz have considered the maximal regularity problem for the case that a linear differential operator $L z=z_{x}-M(x) z$ has an exponential dichotomy on a subinterval $J \subset \mathbb{R}$ (here $M(x)$ are $N \times N$ continuous matrices in $x \in J$ ). In this context the authors have got a similar result to Theorem 3.1.

\section{Maximal regularity for Volterra difference system with infinite delay}

We complete this paper by applying our previous result to the Volterra difference systems with infinite delay. Let $A(n), K(m)$ be $r \times r$ matrices defined for $n \in \mathbb{N}\left(n_{0}\right), m \in \mathbb{Z}^{+}$, and let $\beta: \mathbb{Z}^{+} \rightarrow \mathbb{R}^{+}$be an arbitrary positive increasing sequence such that

$$
\sum_{n=0}^{\infty}|K(n)| \beta(n)<+\infty
$$

We consider the following Volterra difference system with infinite delay

$$
x(n+1)=\sum_{s=-\infty}^{n} A(n) K(n-s) x(s), \quad n \geq n_{0} .
$$

This equation is viewed as a functional difference equation on the phase space $\mathscr{B}_{\beta}$, where $\mathscr{B}_{\beta}$ is defined as follows:

$$
\mathscr{B}_{\beta}=\left\{\varphi: \mathbb{Z}^{-} \longrightarrow \mathbb{C}^{r}: \operatorname{Sup}_{n \in \mathbb{Z}^{+}}|\varphi(-n)| / \beta(n)<+\infty\right\}
$$

with norm

$$
\|\varphi\|_{\mathscr{B}_{\beta}}=\operatorname{Sup}_{n \in \mathbb{Z}^{+}}|\varphi(-n)| / \beta(n), \varphi \in \mathscr{S}_{\beta} .
$$

We have the following result as a consequence of Theorem 3.1.

Theorem 4.1. Assume that system (4.2) has an exponential dichotomy on $\mathbb{N}\left(n_{0}\right)$ with data $(\alpha, \widetilde{K}, P(n))$. Then, for any $h \in \ell^{P}\left(\mathbb{N}\left(n_{0}\right)\right)$ (with $\left.1 \leq p \leq+\infty\right)$ and any $\varphi \in \operatorname{Range}\left(P\left(n_{0}\right)\right)$ the boundary value problem,

$$
\begin{gathered}
z(n+1)=\sum_{s=-\infty}^{n} A(n) K(n-s) z(s)+h(n), \\
P\left(n_{0}\right) z_{n_{0}}=\varphi
\end{gathered}
$$


has a unique solution $z \in \mathcal{W}^{1, p}\left(\mathbb{N}\left(n_{0}\right)\right)$, namely $z=z^{s p}+z^{\text {hom }}$, where

$$
\begin{gathered}
z_{n}^{s p}=\sum_{s=n_{0}}^{\infty} \Gamma(n, s) E^{0}(h(s)), \\
z_{n}^{\text {hom }}=T\left(n, n_{0}\right) P\left(n_{0}\right) \varphi,
\end{gathered}
$$

where $\Gamma(n, s)$ is the Green function associated to (4.2). On the other hand, the solution $z$ satisfies $z \in \ell^{p^{\prime}}\left(\mathbb{N}\left(n_{0}\right)\right)$ for all $1 \leq p \leq p^{\prime} \leq+\infty$ and the following estimates hold:

$$
\begin{gathered}
\left(1-e^{-\alpha}\right)^{1-1 / p+1 / p^{\prime}}\left\|z^{s p}\right\|_{p^{\prime}}+\left(1-e^{-\alpha}\right)^{1-1 / p}\left\|z_{n_{0}}^{s p}\right\|_{\mathscr{B}_{\beta}} \leq 4 K \tilde{K}\|h\|_{p}, \\
\left(1-e^{-\alpha}\right)^{1 / p^{\prime}}\left\|z^{\text {hom }}\right\|_{p^{\prime}}+\left\|z_{n_{0}}^{\text {hom }}\right\|_{\mathscr{\Re}_{\beta}} \leq(\tilde{K}+1)\|\varphi\|_{\mathscr{B}_{\beta}},
\end{gathered}
$$

where $\alpha$ and $\tilde{K}$ are the constants of Definition 2.1(iii)-(iv).

Remark 4.2. Note that in the preceding estimates, we get $1 / p=0$ for $p=+\infty$.

We now want to present an example to illustrate the usefulness of Theorem 4.1.

Example 4.3. Let $a_{i}(n), i=1,2$ be two sequences and $\sigma, \alpha, \gamma$ be three positive constants such that

(i) $\rho_{1}^{*}:=\operatorname{Sup}_{n \geq 0} \max _{-n \leq \theta \leq 0}\left[\left[\prod_{s=n+\theta}^{n-1}\left|a_{1}(s)\right|^{-1}\right] / e^{-\gamma \theta}\right]<+\infty$,

(ii) $\prod_{s=\tau}^{n-1}\left|a_{1}(s)\right| \leq \sigma e^{-\alpha(n-\tau)}, n \geq \tau \geq 0$,

(iii) $\prod_{s=n}^{\tau-1}\left|a_{2}(s)\right|^{-1} \leq \sigma e^{-\alpha(\tau-n)}, \tau \geq n \geq 0$.

Some concrete examples of functions $a_{1}$ and $a_{2}$ satisfying the previous assumptions are

(a) $a_{1}(n):=1 / \delta, a_{2}(n):=\delta$ with $1<\delta \leq e^{\gamma}$ or $1 / \mu \leq \delta \leq v e^{\gamma}$, where $\mu, v \in(0,1)$,

(b) $\eta<e^{-\gamma}<\mu<1, \gamma>0, a_{1}(n):=\mu, a_{2}(n):=1 / \eta$,

(c) $1 / \nu e^{\gamma} \leq\left|a_{1}(n)\right| \leq \mu, 1 / \mu \leq\left|a_{2}(n)\right|$, for all $n \geq 0$, where $\mu, \nu \in(0,1)$.

From now until end of Example 4.3, we will assume that $a_{1}$ and $a_{2}$ are functions satisfying (i)-(iii). Using (ii) and (iii), we can assert that

$$
\prod_{s=\tau}^{n-1}\left|a_{2}(s)\right|^{-1} \leq \sigma^{2} \prod_{s=\tau}^{n-1}\left|a_{1}(s)\right|^{-1}, \quad n \geq \tau .
$$

We consider the following nonautonomous difference system

$$
x(n+1)=A(n) x(n),
$$

where $A(n)$ is a $2 \times 2$ matrix defined by $\operatorname{diag}\left(a_{1}(n), a_{2}(n)\right)$. For convenience of the reader, we would like to begin with a complete analysis to check the dichotomic properties. We recall that the solution operator $T(n, \tau), n \geq \tau$, of (4.9) is a bounded linear operator on 
the phase space $\mathscr{S}_{\beta}$, with $\beta(n)=e^{\gamma n}$, and is defined by

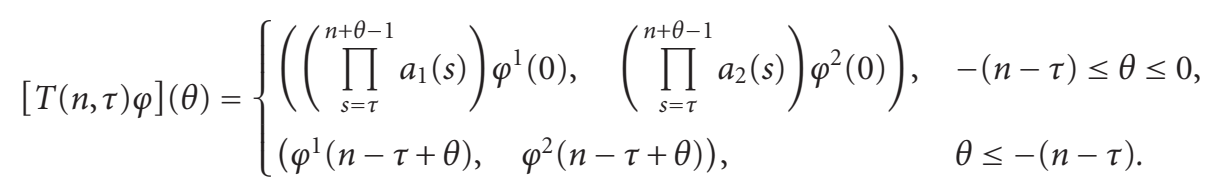

A computation shows that

$$
T(n, s) T(s, m)=T(n, m), \quad n \geq s \geq m, \quad T(m, m)=I .
$$

The problem of deciding when a functional difference system has an exponential dichotomy is a priori much complicated than for ordinary difference system, because it is necessary to construct suitable projections, which play a distinguished role, and also to get some estimates on the norm of solution operator which acts on the phase space with infinite dimension. In our case the projections can be taken as $P(n): \mathscr{B}_{\beta} \rightarrow \mathscr{S}_{\beta}$ given by

$$
[P(n) \varphi](\theta)= \begin{cases}\left(\varphi^{1}(\theta), \varphi^{2}(\theta)-\left(\prod_{s=n+\theta}^{n-1} a_{2}(s)^{-1}\right) \varphi^{2}(0)\right), & -n \leq \theta \leq 0 \\ \left(\varphi^{1}(\theta), \varphi^{2}(\theta)\right), & \theta<-n\end{cases}
$$

and $Q(n)=I-P(n): \mathscr{B}_{\beta} \rightarrow \mathscr{S}_{\beta}$.

For $n \geq \tau$ we observe that $T(n, \tau): Q(\tau) \mathscr{S}_{\beta} \rightarrow Q(n) \mathscr{B}_{\beta}$ is given by

$$
[T(n, \tau) Q(\tau) \varphi](\theta)= \begin{cases}\left(0,\left(\prod_{s=\tau}^{n+\theta-1} a_{2}(s)\right) \varphi^{2}(0)\right), & -(n-\tau) \leq \theta \leq 0, \\ \left(0,\left(\prod_{s=n+\theta}^{\tau-1} a_{2}(s)^{-1}\right) \varphi^{2}(0)\right), & -n \leq \theta \leq-(n-\tau), \\ (0,0), & \theta<-n .\end{cases}
$$

We can see that for $n \geq \tau$,

$$
T(n, \tau) Q(\tau)=Q(n) T(n, \tau), \quad T(n, \tau) P(\tau)=P(n) T(n, \tau) .
$$

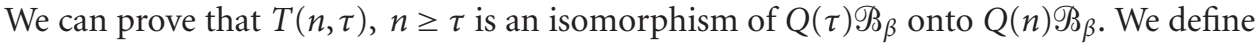
$T(\tau, n)$ as the inverse mapping, which is given by

$$
[T(\tau, n) Q(n) \varphi](\theta)= \begin{cases}\left(0,\left(\prod_{s=\tau+\theta}^{n-1} a_{2}(s)^{-1}\right) \varphi^{2}(0)\right), & -\tau \leq \theta \leq 0, \\ (0,0), & \theta<-\tau .\end{cases}
$$


By virtue of (4.8), we claim that there is positive constant $\widetilde{K}$ such that

$$
\|T(n, \tau) P(\tau)\| \leq \widetilde{K} e^{-\alpha(n-\tau)}, \quad n \geq \tau .
$$

In fact,

$$
\begin{aligned}
\|T(n, \tau) P(\tau)\| \leq & \max _{-(n-\tau) \leq \theta \leq 0}\left[\left[\prod_{s=\tau}^{n+\theta-1}\left|a_{1}(s)\right|\right] e^{\gamma \theta}\right]+3 \max _{-n \leq \theta \leq-(n-\tau)}\left[\left[\prod_{s=n+\theta}^{\tau-1}\left|a_{2}(s)\right|^{-1}\right] e^{\gamma \theta}\right] \\
\leq & {\left[\prod_{s=\tau}^{n-1}\left|a_{1}(s)\right|\right] \max _{-(n-\tau) \leq \theta \leq 0}\left[\left[\prod_{s=n+\theta}^{n-1}\left|a_{1}(s)\right|^{-1}\right] e^{\gamma \theta}\right] } \\
& +3 \sigma^{2}\left[\prod_{s=\tau}^{n-1}\left|a_{1}(s)\right|\right] \max _{-n \leq \theta \leq-(n-\tau)}\left[\left[\prod_{s=n+\theta}^{n-1}\left|a_{1}(s)\right|^{-1}\right] e^{\gamma \theta}\right] \\
\leq & 4 \sigma^{2} \rho_{1}^{*}\left[\prod_{s=\tau}^{n-1}\left|a_{1}(s)\right|\right] .
\end{aligned}
$$

On the other hand, we can verify that

$$
\|T(n, \tau) Q(\tau)\| \leq \sigma \rho_{2}^{*} e^{-\alpha(\tau-n)}, \quad \tau \geq n,
$$

where $\rho_{2}^{*}:=\operatorname{Sup}_{n \geq 0} \max _{-n \leq \theta \leq 0}\left[\left[\prod_{s=n+\theta}^{n-1}\left|a_{2}(s)\right|^{-1}\right] / e^{-\gamma \theta}\right]$.

We note that the projectors $P(n)$ are not unique, but the ranges are unique (see Remark 4.4 for more details). It is worth to note that one can constructing other projectors $\hat{P}(n)$ from $P(n)$ such that (4.9). has an exponential dichotomy. Following the general method established in Remark 4.5 we construct new projectors

$$
[\hat{P}(n) \varphi](\theta)= \begin{cases}\left(\varphi^{1}(\theta)+\left(\prod_{s=0}^{n+\theta-1} a_{1}(s)\right)\left(\prod_{s=0}^{n-1} a_{2}(s)^{-1}\right) \varphi^{2}(0), \varphi^{2}(\theta)\right. & \\ \left.-\left(\prod_{s=n+\theta}^{n-1} a_{2}(s)^{-1}\right) \varphi^{2}(0)\right) & \text { if }-n \leq \theta \leq 0, \\ \left(\varphi^{1}(\theta), \varphi^{2}(\theta)\right), & \text { if } \theta<-n,\end{cases}
$$

such that (4.2) has an exponential dichotomy.

For any $h \in \ell^{P}\left(\mathbb{N}\left(n_{0}\right)\right.$ ) (with $\left.1 \leq p \leq+\infty\right)$ and any $\varphi \in \operatorname{Range}\left(P\left(n_{0}\right)\right.$ ), Theorem 4.1 assure that the boundary value problem

$$
\begin{gathered}
z(n+1)=A(n) z(n)+h(n), \quad n \geq n_{0}, \\
P\left(n_{0}\right) z_{n_{0}}=\varphi,
\end{gathered}
$$

has a unique solution $z \in \mathcal{W}^{1, p}\left(\mathbb{N}\left(n_{0}\right)\right)$. Moreover $z \in \ell^{p^{\prime}}\left(\mathbb{N}\left(n_{0}\right)\right)$ for all $1 \leq p \leq p^{\prime} \leq+\infty$ and the estimates (4.7) hold. 
This finished the discussion of Example 4.3. The next two remark was inspired in a Beyn and Lorenz's appendix about dichotomies (see [3]).

Remark 4.4. In general, the projectors $P(n)$ of an exponential dichotomy are not unique. (Of course, by Definition 2.1(i) and (ii), if a projector $P(m)$ is determined at one point $m$, then all projectors $P(n)$ are determined uniquely.) However the ranges are unique because they can be written as

$$
\operatorname{Ranges}(P(m))=\left\{\varphi \in \mathscr{B}: e^{-\eta(n-m)} T(n, m) \varphi \text { is bounded for } n \geq m\right\}
$$

for any $0<\eta<\alpha$. While " $\subseteq$ " is obvious the converse conclusion follows from

$$
\|Q(m) \varphi\|_{\mathscr{B}}=\|T(m, n) Q(n) T(n, m) \varphi\|_{\mathscr{B}} \leq C e^{(\alpha-\eta)(m-n)} \longrightarrow 0, \quad \text { as } n \rightarrow \infty,
$$

where $C$ is a suitable constant.

Remark 4.5. Let $(\alpha, \tilde{K}, P(n))$ be the data of an exponential dichotomy. Now we turn our attention to the following question: how one can constructing other projectors $\widehat{P}(n)$ from $P(n)$ such that (1.1) has an exponential dichotomy? The answer is: take a projector $\hat{P}\left(n_{0}\right)$ that satisfies Range $\left(\hat{P}\left(n_{0}\right)\right)=\operatorname{Range}\left(P\left(n_{0}\right)\right)$. This allows us to define the following projectors:

$$
\widehat{P}(n)=P(n)+T\left(n, n_{0}\right) \hat{P}\left(n_{0}\right) T\left(n_{0}, n\right) Q(n) .
$$

Then (1.1) has an exponential dichotomy with data $\left(\alpha, \widetilde{K}_{\#}, \widehat{P}(n)\right)$, where $\widetilde{K}_{\#}=\widetilde{K}+$ $\widetilde{K}^{2}|| \hat{P}\left(n_{0}\right) \|$.

\section{References}

[1] W. Arendt and S. Bu, The operator-valued Marcinkiewicz multiplier theorem and maximal regularity, Mathematische Zeitschrift 240 (2002), no. 2, 311-343.

[2] W.-J. Beyn and J. Lorenz, Stability of traveling waves: dichotomies and eigenvalue conditions on finite intervals, Numerical Functional Analysis and Optimization 20 (1999), no. 3-4, 201-244.

[3] __ Stability of viscous profiles: proofs via dichotomies, preprint, 2004.

[4] S. Blunck, Analyticity and discrete maximal regularity on $L_{p}$-spaces, Journal of Functional Analysis $\mathbf{1 8 3}$ (2001), no. 1, 211-230.

[5] Maximal regularity of discrete and continuous time evolution equations, Studia Mathematica 146 (2001), no. 2, 157-176.

[6] C. Cuevas, Weighted convergent and bounded solutions of Volterra difference systems with infinite delay, Journal of Difference Equations and Applications 6 (2000), no. 4, 461-480.

[7] C. Cuevas and L. Del Campo, An asymptotic theory for retarded functional difference equations, Computers \& Mathematics with Applications 49 (2005), no. 5-6, 841-855.

[8] C. Cuevas and M. Pinto, Asymptotic behavior in Volterra difference systems with unbounded delay, Journal of Computational and Applied Mathematics 113 (2000), no. 1-2, 217-225.

[9] Asymptotic properties of solutions to nonautonomous Volterra difference systems with infinite delay, Computers \& Mathematics with Applications 42 (2001), no. 3-5, 671-685.

[10] Convergent solutions of linear functional difference equations in phase space, Journal of Mathematical Analysis and Applications 277 (2003), no. 1, 324-341. 
[11] C. Cuevas and C. Vidal, Discrete dichotomies and asymptotic behavior for abstract retarded functional difference equations in phase space, Journal of Difference Equations and Applications 8 (2002), no. 7, 603-640.

[12] S. Elaydi, S. Murakami, and E. Kamiyama, Asymptotic equivalence for difference equations with infinite delay, Journal of Difference Equations and Applications 5 (1999), no. 1, 1-23.

[13] J. K. Hale and J. Kato, Phase space for retarded equations with infinite delay, Funkcialaj Ekvacioj 21 (1978), no. 1, 11-41.

[14] Y. Hino, S. Murakami, and T. Naito, Functional-Differential Equations with Infinite Delay, Lecture Notes in Mathematics, vol. 1473, Springer, Berlin, 1991.

[15] V. B. Kolmanovskii, E. Castellanos-Velasco, and J. A. Torres-Muñoz, A survey: stability and boundedness of Volterra difference equations, Nonlinear Analysis 53 (2003), no. 7-8, 861-928.

[16] H. Matsunaga and S. Murakami, Some invariant manifolds for functional difference equations with infinite delay, Journal of Difference Equations and Applications 10 (2004), no. 7, 661-689.

[17] S. Murakami, Representation of solutions of linear functional difference equations in phase space, Nonlinear Analysis. Theory, Methods \& Applications 30 (1997), no. 2, 1153-1164.

[18]__ Some spectral properties of the solution operator for linear Volterra difference systems, New Developments in Difference Equations and Applications (Taipei, 1997), Gordon and Breach, Amsterdam, 1999, pp. 301-311.

[19] L. Weis, A new approach to maximal $L_{p}$-regularity, Evolution Equations and Their Applications in Physical and Life Sciences (Bad Herrenalb, 1998), Lecture Notes in Pure and Appl. Math., vol. 215, Marcel Dekker, New York, 2001, pp. 195-214.

[20] _ Operator-valued Fourier multiplier theorems and maximal $L_{p}$-regularity, Mathematische Annalen 319 (2001), no. 4, 735-758.

Claudio Cuevas: Departamento de Matemática, Universidade Federal de Pernambuco,

CEP. 50540-740 Recife-PE, Brazil

E-mail address: cch@dmat.ufpe.br

Claudio vidal: Departamento de Matemática, Universidade Federal de Pernambuco,

CEP. 50540-740 Recife-PE, Brazil

E-mail address: claudio@dmat.ufpe.br 